le portiQue Le Portique

Revue de philosophie et de sciences humaines

$36 \mid 2016$

\title{
Michel Leiris
}

\section{Allégorie, détails et digression}

Allegory, details and digression.

Allegorie, Details und Digression.

Jean-François Poirier

\section{OpenEdition}

Journals

Édition électronique

URL : http://journals.openedition.org/leportique/2853

DOI : $10.4000 /$ leportique.2853

ISSN : $1777-5280$

Éditeur

Association "Les Amis du Portique"

Édition imprimée

Date de publication : 15 février 2016

ISSN : 1283-8594

\section{Référence électronique}

Jean-François Poirier, « Allégorie, détails et digression », Le Portique [En ligne], 36 | 2016, document 8, mis en ligne le 15 février 2017, consulté le 26 mars 2021. URL : http://journals.openedition.org/ leportique/2853 ; DOI : https://doi.org/10.4000/leportique.2853

Ce document a été généré automatiquement le 26 mars 2021.

Tous droits réservés 


\title{
Allégorie, détails et digression
}

\author{
Allegory, details and digression. \\ Allegorie, Details und Digression.
}

Jean-François Poirier

1 Je me suis imprudemment engagé à remettre un texte au Portique, revue qui m'est chère et qui s'est toujours montrée très hospitalière à mon égard, sur Michel Leiris à l'occasion d'un numéro spécial que cette revue lui consacre. Bien mal m'en a pris, car en me replongeant dans l'œuvre de cet auteur, que j'ai lue presque intégralement au cours de l'année que j'ai passée à Berlin en 1975-1976 comme assistant de langue à la Georg-Herwegh-Schule, force me fut de reconnaître que non seulement la ferveur qu'avait suscitée en moi cet auteur n'était plus là mais aussi qu'elle avait laissé la place à une gêne fortement teintée de déplaisir. J'ai donc lu Leiris à Berlin en 75-76, durant un hiver glacial, je l'ai aimé à la passion, d'une passion froide et triste. Le Berlin de cette époque, Jean-Michel Palmier en a donné une bonne évocation dans Berliner Requiem, le livre est plein d'emphase, de grandiloquence, mais nous l'étions aussi et il a bien capté l'effervescence stérile que connaissait alors la ville qui agençait dans mon esprit, et dans celui de bien d'autres j'imagine, un montage qui faisaient tenir ensemble une ville gâtée par les subventions de l'Ouest, un revival des années folles de la République de Weimar, le spectre du III ${ }^{e}$ Reich et une version pétrifiée du communisme. Leiris avait fusionné pour moi avec le lieu où je l'ai lu. Il a coulé beaucoup d'eau froide dans ma tête (alors bouillonnante) depuis ce temps-là, pour reprendre une expression que Leiris emploie dans La Règle du jeu. Je ne vais plus à Berlin. Je préfère aujourd'hui infiniment à Leiris Marcel Jouhandeau, diariste souvent filou mais qui a su créer sinon un mythe pour notre temps, du moins un mythe local qui n'est pas sans saveur, qui a été un ami de jeunesse de Leiris et, à ce que je sais, l'est resté, avec une certaine distance, jusqu'à la fin. Jouhandeau cultivait l'amour de soi, différent, et même à l'opposé, de l'amourpropre (mauvais car complaisant), comme le dit très bien Rousseau. J'ai longtemps cru que pour échapper à l'amour-propre il fallait être méchant avec soi-même, ce que Leiris était notoirement. C'est en fait très insuffisant, il ne sert à rien de faire la guerre au mal, fût-il en soi, ou, pour dire les choses plus prudemment, cela ne garantit pas qu'on ait emprunté la voie du bien. 
2 Je viens de relire L'Ennemi déclaré de Genet et je vois qu'il situe en 75/76 la coupure entre une époque qui croit en l'action collective et une époque qui n’y croit plus ( $/ \mathrm{Je}$ suis un porc, et le porc rentre chez lui ", La Noce chez les petits bourgeois, Brecht), deux années où beaucoup de choses ont changé dans ma vie, si bien qu'il m'est difficile de faire la différence entre ce qui a changé pour moi et ce qui a changé dans l'époque. Maintenant je suis certain que c'est bien l'époque qui a changé au cours de ces deux années. Ne voulant pas abuser de l'hospitalité de mes hôtes en me conduisant mal envers l'auteur qu'ils souhaitent honorer ni non plus me lancer dans un panégyrique dont l'insincérité et l'inauthenticité aurait été honteuses ni encore leur faire faux bond, j'ai finalement décidé de me rallier à la solution que m'a obligeamment suggérée le directeur de la publication en procédant "per via di levare », expression dont j'ai vérifié le sens car mon goût de l'histoire de l'art s'est éteint il y a un peu plus de deux décennies et je n'avais plus qu'une idée assez vague des théories artistiques de la Renaissance dont relève le per via di levare. Cette idée me plaît que l'œuvre, ici un modeste article, est enserrée dans un bloc de matière, une gangue, et qu'il suffit de procéder par retrait de la matière qui la dissimule aux regards pour faire apparaitre ce que je qualifierais d'«Idea ", par référence à mon historien de l'art préféré Erwin Panofsky (curieusement oublié au profit d'Aby Warburg qui, quant à moi, me parait un original mais nullement un maître de l'histoire de l'art comme Panofsky), pour désigner (peut-être abusivement) l'insécable, comme on dit en traitement de texte, de mon propos.

3 J'ai accepté, ne sachant pas à quels tourments j'allais m'exposer. Ne retirer ni trop ni trop peu de matière. Trop et c'est le cœur qui est endommagé, trop peu et celui-ci apparaît informe, comme embourbé, plus semblable à une motte de glaise qu'à une statue. Je trouve toujours le titre de mes textes avant de les écrire et cette fois j'hésite, j'avais pensé à "Le bon Dieu est dans les détails", par référence au texte d'une excellente conférence de Jean-Loup Thébaud dont le texte a été publié dans cette revue et qui s'intitulait "Le diable est dans les détails", une formule qu'on a coutume d'attribuer à Aby Warburg «Der Teufel steckt im Detail » alors qu'elle appartient au fonds de la langue allemande sans qu'une attribution à un auteur paraisse possible, les deux expressions étant employées indifféremment avec des sens qu'on peut dire équivalents dans leur valorisation du minuscule, du détail, de l'insignifiant dont on sait bien qu'il n'existe pas pour Dieu, tout étant d'égale importance pour Lui. Dans cet examen attentif et minutieux des choses, à ce qu'elles ont de plus insignifiant, Walter Benjamin reconnaissait la grandeur morale de Proust qui avait, par la profusion des détails bourgeonnant dans l'éternel printemps d'un homme malade et vieillissant, témoigné de sa fidélité aux plus humbles choses. Benjamin avait tenté d'en convaincre le grand germaniste Félix Bertaux, auteur avec Lepointe du célèbre dictionnaire éponyme, alors que, plus philologue qu'artiste, Bertaux résumait la démarche de Proust en ces termes : «Un morceau de fromage sous un microscope ». Marie-Agnès Barathieu rappelait dans un ouvrage passé injustement inaperçu, Les Mobiles de Proust, que "l'un des trois titres que Proust en 1913 encore envisageait de donner à l'un des trois volumes de la Recherche " était L'Âge des choses. J'aurais pu ainsi développer, à la suite de ce titre, que La Règle du jeu participe de cette démocratie littéraire que Jacques Rancière a théorisée. Yvetot vaut Constantinople. Mais je ne suis pas sûr que cette fidélité soit ce qui caractérise le mieux Leiris. 
Quelques semaines de macération plus tard, j'en suis arrivé à penser que ce dont témoigne le plus l'œuvre de Leiris est de la progression générale de la mort, d'une nécrose dans l'art et la civilisation en général. Ce n'est guère original, « Le mort saisit le vif ", est un adage juridique auquel Marx a fait un sort philosophique fameux, en le hissant au rang de Zeitdiagnose, il y a bien longtemps, mais c'est un fil conducteur qui me paraît mener un peu plus loin, un tout petit peu plus loin que mon idée première, sans que je sache vraiment dans quelle direction. Mon étude de l'œuvre de Simmel à laquelle je me suis consacré depuis quelques années m'a permis d'y voir plus clair, notamment grâce au grand livre de Lilyane Deroche-Gurcel sobrement intitulé Simmel et la modernité. Disons que le choix capital, entre deux positions extrêmes, qui s'est présenté à la littérature (et l'art en général) depuis un temps que je ne saurais trop déterminer (un siècle ou plutôt deux) me paraît avoir été entre l'allégorie et le réenchantement du monde. Le choix allégorique (mais est-ce bien un choix ?) consiste à prendre le monde présent comme les membra disjecta d'un torso qu'il faudrait remembrer pour lui restituer son intégrité, mais une intégrité autre que celle que possédait la statue avant qu'elle ne soit brisée. Un peu de latin sied à Leiris, je recopie sur Internet :

"Cette expression dériverait de l'expression disjecti membra poetae (littéralement un poète en ses membres épars), issue d'un texte du poète Horace, tiré de ses Satires (Satire IV ${ }^{\mathrm{e}}$.

Ce texte dit en Latin ce qui suit :

[...] His ego quae nunc, Olim quae scripsit Lucilius, eripias si Tempora certa modosque, et quod prius ordine verbum est Posterius facias, praeponens ultima primis : Non (ut si silvas, « Postquam Discordia tetra Belli ferratos postes portasque refregit ») Invenias etiam disjecti membra poetae.

6 Ce que Pierre Daru traduit magnifiquement en vers de la façon suivante :

Essayez de soumettre à cette épreuve utile Et les vers que je fais et les vers de Lucile ; Le poète s'éclipse et ne s'y trouve plus. Au contraire, entendez la Muse d'Ennius : "Quand de son bras d'airain, si fatal à la terre, La Discorde eut brisé les portes de la guerre"; Détruisez l'harmonie, et renversez les mots, Vous y verrez toujours un poète en lambeaux. »

7 Voir http://www.nomina-nuda-tenemus.fr/index.php/premier-jour/sexte-premierjour/52-disjecta-membra

8 Que la solution allégorique soit périlleuse, la Vénus d'Ylle et son "Cave amantem » nous en instruit. La lecture de Baudelaire et sa biographie achèvent de nous en convaincre.

9 La solution du réenchantement paraît, quant à elle, à l'opposé, euphorique. Ce fut, on le sait, la solution choisie par Rilke.

10 «Inspiré par la beauté des paysages et par la profonde spiritualité du peuple russe, qu'il découvre lors d'un voyage en 1900, Rilke est convaincu de la présence de Dieu à chaque 
instant de sa vie et dans chacun de ses gestes, "sanctifiant" son travail. C'est cette conviction qu'illustrent les nouvelles de ce recueil. Ces Histoires du Bon Dieu s'inspirent à la fois des contes universels et de la chanson populaire russe, "ces mélodies, écrit Rilke, que personne, fût-il cosaque ou paysan, n'a jamais pu entendre sans pleurer". Le poète interprète librement le récit biblique de la création du monde et le martyre du Christ, retrace la rencontre du tsar Ivan Vassiliévitch avec un bâtisseur d'églises qui pourrait être Dieu, relate le retour de Jego, fils exilé, auprès de son père, et le destin d'un vieux peintre d'icônes ou une scène du ghetto de Venise ». Ainsi est présentée par son éditeur l'œuvre de Rilke, Histoires du Bon Dieu, qui passe pour donner la formule de la solution résurrective : je meurs, mais c'est pour ressusciter en Dieu.

Baudelaire et Rilke, Cocteau et les surréalistes, Giraudoux et Céline (le réenchantement est chez lui pour une autre fois), les premiers ont choisi l'allégorie, les seconds le réenchantement. Si le lecteur me permet cette incise, je n'avais pas été peu étonné d'entendre Jacques Rancière dans un débat d'après conférence dire que le poète capital de sa jeunesse avait été Rilke. Qui parait être plus éloigné de la démocratie littéraire que l'auteur des Duineser Elegien? Mais ce goût qui avait été le sien (et qui ne l'était peut-être plus) me semble cohérent avec son absence d'intérêt pour l'allégorie. Pour lui il n'y a pas de modernité mélancolique, peut-être pas de modernité du tout au sens d'une coupure, d'une période et rien ne s'étant arrêté, rien n'étant mort, aucun nouveau départ, aucune résurrection ne doivent être espérés. Tout continue.

Leiris tient, à n'en pas douter, pour la solution allégorique. Le monde est mort, non seulement la vie ne vit pas (Adorno) mais la mort est elle-même morte, telle pourrait être la formule alchimique de l'allégorèse. "J'ai toujours été séduit par les allégories ", écrit-il dans L'Âge d'homme. Qui est-il ? "Un être en qui la fatalité se conjugue avec sa volonté, et qui semble obliger le sort à lui façonner sa statue ». Ce devenir-statue est le fait de sa soumission et de sa volonté, laquelle a été supérieurement exaucée par la monumentale édition de la Pléiade. Sa fascination pour Rome est extrême. Il en aime la statuaire alors que la grâce grecque le laisse insensible, il est attiré par les ruines imposantes que les Romains nous ont laissées, il se voit lui-même comme un sénateur romain (il a eu très tôt eu une tête de pierre usée comme on en voit dans les musées du Capitole) participant peu aux affaires publiques, assistant avec dégoût aux jeux du cirque mais trop lâche pour marquer de son absence sa désapprobation.

Dans cette longue autobiographie qu'est $L a$ Règle du jeu, certains voient un mouvement baroque de spirale où tout diffère en se répétant, où tout se répète en différant, je suis plutôt tenté d'y voir une immense fresque où il procède au collationnement infini d'un original vivant mais inaccessible et du masque mortuaire de celui-ci, qui seul nous est parvenu. Il y a chez lui une obstination sidérante à réitérer ce constat que les choses mortes sont incapables de nous faire imaginer ce qu'elles étaient au temps où elles étaient vivantes, sit venia verbo. Roubaud reprochait à Leiris d'avoir voulu reconstituer des manières de pensée alors qu'il n'est, selon lui, possible que de ramener des eaux de la mémoire, dans les filets lancés par l'homme qui se retourne vers son passé, que des coquilles vides et quelques objets flottant après un naufrage. Ce dont l'oulipien lui fait grief est peut-être ce qu'il y a de plus grand chez Leiris, cette obstination à plonger et à plonger encore alors que ce qu'il ramène des fonds pélagiques ressemble de moins en moins aux pièces d'un trésor immergé mais de plus en plus à des détritus marins qui risquent de faire disparaître l'Albatros, espèce menacée, dit-on, par la consommation des « déchets en mer». 

Orient, d'un Autre, qui anime la recherche de Leiris, si on le réduit à sa plus simple expression, à sa formulation la plus sobre peut se résumer dans le mot de « digression ». La digression fait craquer, exploser le carcan de la forme classique mais ne va nulle part, n'annonce aucune forme nouvelle, elle est bien davantage le signe d'une désagrégation universelle, d'une dissolution de tout. Elle est cette pression qu'exerce la lave de l'imagination sur la croûte du style, de la forme. Elle est cette pression qu'exercent les barbares sur le limes. Elle n'est pas un procédé, ou bien alors au sens que ce mot a quand il désigne l'extrême pointe de la queue du billard qui communique son mouvement à la boule. Elle est dunamis et non energeia. On sait qu'Edgar Allan Poe n'aimait pas Montaigne parce qu'il digressait et n'enserrait pas sa pensée dans une forme close. Baudelaire a dû aimer cette rigueur chez Poe, Baudelaire n'aime pas la digression, il ne s'éloigne jamais du point présent qui est un point mort pour embarquer, comme Hugo, le lecteur vers les hautes eaux de l'éternité (aucune mention chez Baudelaire de l'avenir qui est tabuisiert, note Benjamin), il l'a même peutêtre tellement aimée cette rigueur, qu'il lui a donné à sa traduction de Poe une forme plus formelle que celle de l'original en langue américaine, si j'en crois le traducteur et américaniste Jacques Debouzy que j'ai entendu dire à propos des proses d'Edgar Poe que Baudelaire leur avait donné un poli, une perfection qu'elles étaient loin d'avoir dans la langue d'origine (ce dont je ne peux juger par moi-même, n'étant pas angliciste). Baudelaire a peut-être voulu par cette infidélité bienveillante davantage que rapprocher, par une conversion à un certain classicisme, Poe du goût français, plutôt transposer dans la langue l'élément logique si obsédant chez Poe qui n'était pas incompatible pour l'auteur avec une certaine négligence dans l'exposition ni corruptible par un certain relâchement de syntaxe.

Baudelaire a sabré beaucoup de digressions en traduisant Les Confessions d'un mangeur d'opium de De Quincey (voir «La digression chez Baudelaire et De Quincey » de Janis Locas). Les amateurs de littérature anglaise (dont je ne suis pas) tiennent De Quincey pour un maximaliste de la digression, poussant celle-ci au-delà des limites du supportable. Mais pour les amateurs de littérature française (dont je suis), c'est Proust, qui emporte toutes les digues de la phrase, inonde toutes ses berges, il est la digression faite écrivain, toute son œuvre n'est qu'une longue digression depuis un point qu'on a depuis longtemps (toujours déjà, immer schon, comme disent les Allemands, expression qu'il n'est pas facile de traduire sans éprouver un léger sentiment de ridicule) perdu de vue dès qu'on est embarqué sur sa nef.

La digression crée du lien. Elle crée du lien (et peut-être de la vie, si la vie est le continuum des unités discrètes où celles-ci se fondent si bien qu'aucun point du temps ne peut être saisi, mis de côté pour être réemployé plus tard), elle est consolatrice et aux antipodes du très peu consolateur auteur de « Ligeia », chez qui le réenchantement prend la figure d'une atroce réincarnation.

On s'est souvent gaussé du premier lecteur de Proust, Jacques Madeleine, qui écrivait dans son rapport de lecture en 1912 pour les éditions Fasquelle : « Pendant des heures... à propos de ce verre, ou de la bataille de Waterloo... il vous envoie chemin faisant des choses auxquelles on n'aurait jamais pensé... c'est-à-dire des choses qui, cela est très juste, ne sont pas quelconques, sont nouvelles, fines, pleines d'observation et de pénétration, mais qui vous sont envoyées pendant des heures et chemin faisant, c'est-àdire sans que l'on voie jamais où ce chemin conduit. » De même pour le critique Paul 
Souday qui voyait dans le premier volume paru de la Recherche « une surabondance de menus faits ». Dans l'enfer des zoïles on rencontre également Henri Ghéon regrettant que Proust refuse au lecteur «cette satisfaction organique, que nous procure une œuvre dont nous embrassons d'un regard tous les membres, la forme ». Il faut prendre très au sérieux les écrivains qui ont critiqué Proust et ne pas se contenter de penser qu'ils ont raté le coche. Je ne suis pas certain qu'il soit bon d'être consolateur, comme le sont les digressions de Proust ou les envolées lyriques d'Hugo qui sont l'équivalent prospectif et prophétique de l'Odyssée proustienne. N'avons-nous pas davantage besoin aujourd'hui d'intelligence et de courage que de consolations, d'intelligence courageuse et de courage intelligent? Pour ce qui est des exemples de courage (mais peut-être devait-on alors parler d'intrépidité) sans intelligence, les journaux, Internet, les rues en regorgent. Peut-être Mallarmé, que Barthes disait être l'un des seuls à toujours rester maitre de sa peau, jamais sujet à des « empourprements stupides ", a-t-il tenu les deux rênes, mais pour qu'il brille de nouveau, il faudrait d'abord éteindre les deux interprétations qui, au $x^{e}$ siècle, l'ont éclairé en sa vitrine, la version accoudée à la cheminée de Valéry et la version maoïste que des cercles intellectuels français en ont donnée dans les années 1970. Mao s'est arrêté, Mallarmé pourrait reprendre sa longue marche, et tant pis pour la Théorie du sujet de Badiou.

18 Leiris s'est à plusieurs reprises explicitement référé à Proust pour dire toute l'admiration que son œuvre suscite en lui. «... Je trouve Proust génial. Dans peu d'ouvrages, il m'est arrivé à tel point de trouver ce que j'ambitionnais moi-même de faire». "Bien entendu, Proust m'a inspiré dans ma démarche». Mais la grande différence entre Proust et Leiris tient à la langue. «Il est difficile, quand on est troublé par les idées de Kant et la nostalgie de Baudelaire, d'écrire le français exquis d'Henri IV », écrit Proust. "Ce volume si long ne se lit point aisément. Il est non seulement compact, mais obscur. Cette obscurité, à vrai dire, tient moins à la profondeur de la pensée qu'à l'embarras de l'élocution. [...] Ajoutez que les incorrections pullulent, que les participes de M. Proust ont, comme disait un personnage de Labiche, un fichu caractère, en d'autres termes qu'ils s'accordent mal ; que ses subjonctifs ne sont pas plus conciliants ni plus disciplinés, et ne savent même pas se défendre contre les audacieux empiètements de l'indicatif ». Ce jugement négatif de Paul Souday ne pourrait en aucun cas s'appliquer à Leiris dont l'hypercorrection grammaticale est sans défaut.

19 Ce qui fait que la langue de Proust et la langue de Leiris ne se ressemblent pas du tout, c'est le recours à la syllepse chez le premier. La syllepse donne la fluidité de la pensée à langue, elle ne se soucie pas du bon usage, elle traite cavalièrement la grammaire, enjambe les haies syntaxiques. Je me souviens qu'enfant nous avions reçu par la poste une édition à compte d'auteur d'un recueil de poèmes d'une vague cousine. Un de ces poèmes s'intitulait Mont Saint-Michel et on y trouvait un vers dont ne surnage dans ma mémoire que ces mots «boulonné sur son socle rocheux " et j'entendais mon père murmurer, non ce n'est pas possible, on ne peut dire cela. Et bien chez Leiris tout est boulonné sur son socle rocheux. La phrase proustienne file comme une fusée, poursuivie par elle-même, elle impose au lecteur d'aller dans sa lecture aussi vite qu'elle. Pas de temps perdu à une reconstitution archéologique. Tous ceux-là me comprendront que parfois le vent en passant a soudain touchés du désir irrésistible de fuir avec lui jusqu'à la mer où ils pourront voir, au lieu des inertes pavés du village vainement cinglés par la tempête, les flots soulevés, lui rendre coup pour coup et rumeur pour rumeur (Phrase de 
Proust dans un article sur un voyage en automobile si peu correcte que mes lecteurs du Portique voulaient la corriger).

\section{RÉSUMÉS}

Leiris est-il un allégoricien?

Leiris est-il un néoclassique malgré son usage de la digression?

Leiris écrit-il dans une langue morte ou dans une langue vivante?

Is Leiris an allegory maker?

Is Leiris neoclassical despite his use of digression?

Does Leiris write in a dead language or a living tongue?

Schreibt Leiris Allegorien? Ist er ein Neoklassiker, selbst wenn er zur Digression greift? Ist Leiris Sprache eine tote Sprache oder eine lebendige?

\section{AUTEUR}

\section{JEAN-FRANÇOIS POIRIER}

Jean-François Poirier, né le 17 février 1951 à Soisy-sous-Montmorency, est germaniste et romaniste. Il a publié Bréviaire de la vie de chez soi I (2010) et La Maison du Tremblay (2013) chez Fage Éditeur. 